\title{
Diameter-Extremal Subsets of Spheres
}

\author{
Mikhail Katz* \\ Department of Mathematics, State University of New York, Stony Brook, NY 11794, USA
}

\begin{abstract}
We investigate those spherical point sets which, relative to the Hausdorff metric, give local minima of the diameter function, and obtain estimates which, in principle, justify computer-generated configurations. We test the new sets against two classical conjectures: Borsuk's and McMullen's.
\end{abstract}

\section{Introduction}

It is well known that the vertices of a regular tetrahedron define the "best" way of placing four points on the circumscribed sphere in the sense that the minimal distance among points is as big as possible (see p. 114 of [4]). It is less known that the same configuration also has the "dual" property in the sense that it locally minimizes the maximal distance among points, i.e., no nearby set has a smaller diameter. This paper proposes to study the class of finite subsets which possess such an extremal property.

Let $Y \subset S^{n}$ be a finite subset containing no opposite points of the unit sphere $S^{n}$. Let $d=\operatorname{diam} Y=\max _{x, y_{\in} \in Y} \operatorname{dist}(x, y)$. Let $y \in Y$. Denote by yz the unit tangent vector at $y$ to the shortest arc joining $y$ with $z$. Let $x$ run through all points of $Y$ such that $\operatorname{dist}(x, y)=d$. We say that a point $y \in Y$ is held by $Y$ if the following two equivalent conditions are satisfied:

1. For every tangent vector $v \in T_{y} S^{n}$, there is a point $x$ such that $v \cdot \mathbf{y x} \geq 0$.

2. For some $a_{x} \geq 0$, not all zero, $\sum a_{x} \mathbf{y x}=0$, where the summation is taken over all $x$ as above.

Definition. A set $Y \subset S^{n}$ is called pointwise extremal if every point $y \in Y$ is held by $Y$.

\footnotetext{
* Supported in part by NSF Grant DMS 8602645.
} 
It is not entirely obvious that such sets actually exist, so here are two examples of pointwise extremal sets:

1. The set of vertices of a regular $(2 k+1)$-gon inscribed in the circle $S^{\prime}$.

2. The set of vertices of a regular $(n+1)$-simplex inscribed in $S^{n}$.

More examples can be obtained inductively on dimension by the pyramid construction. Suppose $Y \subset S^{n-1}$ is pointwise extremal. We view $Y$ as a subset of $R^{n+1}$ by imbedding $S^{n-1} \subset R^{n} \subset R^{n+1}$. Let $\operatorname{diam}_{\mathrm{E}} Y=\max _{w ; *} y\|x-y\|$ be the Euclidean diameter of $Y$. Choose $p \in R^{n+1}$ so that, for every $y \in Y \subset R^{n+1}$, one has $\|p-y\|=\operatorname{diam}_{E} Y$. Then the set $Y \cup\{p\}$, scaled by a suitable factor, is inscribed in a unit sphere $S^{n}$ and is a pointwise extremal subset of $S^{n}$ (pyramid over $Y$ ).

In the two-dimensional case we have the following.

Proposition 1. Pointwise extremal subsets of $S^{2}$ include two infinite families of subsets with, respectively, $2 k+2$ and $3 k+1$ points for every $k \geq 1$. Each of these subsets has diameter less than $2 \pi / 3$.

Here the diameter of $Y \subset S^{2}$ is measured in terms of spherical distances, i.e., lengths of arcs of great circles.

Remark. If $Y \subset S^{2}$ is pointwise extremal then it is clear that $Y$ lies in no open hemisphere, hence diam $Y \geq \arccos \left(-\frac{1}{3}\right)$ with equality only for the set of vertices of a regular inscribed tetrahedron (see [14]).

Theorem 1. Suppose $Y \subset S^{2}$ is a pointwise extremal set with $n$ points and with $\operatorname{diam} Y=d<2 \pi / 3$. Then the number of pairs of points $\{x, y\}$ in $Y$ with $\operatorname{dist}(x, y)=d$ equals $2 n-2$.

An antiprism over a square such that the diagonal of the square equals the distance from a top vertex to the two farthest bottom vertices, has eight vertices and only 12 maximal distances. Thus the diameter restriction is indispensable.

An analog of Theorem 1 also holds for sets which are approximately pointwise extremal (a suitable definition is given in 4.6). This observation is the key to justifying computer-generated examples.

The proof of Theorem 1 will actually imply the following: the Euclidean convex hull of $Y \subset R^{3}$ is a polyhedron which is dual to itself (up to homothety). Such objects were considered by Lovász [11].

The proof of Theorem 1 has a similar flavor to the proofs of Borsuk's conjecture for finite subsets of $R^{3}$ (see [2], [8], [9], and [16]). Restricting the class of subsets to subsets of the sphere actually enlarges the family of known examples. In fact, such a restriction enables one to apply a procedure of successive approximation to construct new sets (see Section 2). However, we found no counterexample to the conjecture for $R^{4}$ among a few pointwise extremal subsets of $S^{3}$ (see also Section 5).

Next we define the space $D\left(S^{n}, c\right)$, where $c>0$, of all finite subsets $Y \subset S^{n}$ with diam $Y<c$. The diameter function, defined on $D\left(S^{n}, c\right)$, associates to every $Y \in D\left(S^{n}, c\right)$ its diameter diam $Y \subset S^{n}$. The Hausdorff distance dist ${ }_{H}$ defines a metric on $D\left(S^{n}, c\right)$. 
A set $Y \in D\left(S^{n}, c\right)$ is called a critical point of the diameter function if

$$
\operatorname{diam} Y<\operatorname{diam} Y^{\prime}+o\left(\operatorname{dist}_{H}\left(Y, Y^{\prime}\right)\right)
$$

for all $Y^{\prime} \subset S^{n}$. Equivalently, one may define a critical point $Y$ by saying that small perturbations of $Y$ can only decrease diam $Y$ quadratically in the size of the perturbation.

Similarly, $Y \in D\left(S^{n}, c\right)$ is called a minimum of the diameter function (or, briefly, a minimal set) if diam $Y \leq \operatorname{diam} Y^{\prime}$ for all sufficiently small perturbations.

Theorem 2. Every critical point of the diameter function on $D\left(S^{2}, 2 \pi / 3\right)$ is a minimal set on $S^{2}$.

Note that the number $2 \pi / 3$ in Theorem 2 is the best possible, as the set of vertices of an equilateral triangle inscribed in a great circle has diameter $2 \pi / 3$ and defines a critical point of the diameter function which is not a minimal set.

Corollary 1. If $d<2 \pi / 3$, there are only finitely many noncongruent minimal subsets of $S^{2}$ with diameter $\leq d$.

We may view the diameter function as a "Morse function" on the space of subsets. A Morse theory-type argument yields the following (see Section 6).

Corollary 2. There is exactly one (up to congruence) minimal set in each connected component of $D\left(S^{2}, 2 \pi / 3\right)$.

Question 1 (due to U. Abresch). Given that a minimal set is always pointwise extremal (Corollary 3.4 ), is the converse also true?

In Section 1 we describe a general construction of pointwise extremal sets and prove Proposition 1. Section 2 introduces a notion of separability and explains how to use the sets of the previous section to generate new examples by computer. In Section 3 we study the tiling of $S^{2}$ defined by a pointwise extremal set of diameter $<2 \pi / 3$, and prove Theorem 1 . Section 4 extends Theorem 1 to approximately pointwise extremal sets.

Section 5 describes a way of rewriting an inequality due to Walkup [17]. This inequality is a special case of McMullen's conjecture [12, p. 561], proved in [15] and [1]. It relates the numbers of edges and vertices of a convex simplicial 4-polytope. We rewrite this inequality in the context of self-dual polytopes defined by pointwise extremal subsets of $S^{3}$, and observe that the rewritten version involves the number of pairs of vertices at maximal distance.

In Section 6 we prove Theorem 2 essentially by counting the degrees of freedom. Section 7 provides sufficient conditions for the existence of a minimal set near an approximately minimal set. 
I came to consider minimal sets while thinking of $M$. Gromovs notion of the Filling Radius (see [6] and [10]). To sum up briefly the connection between the two, one could conjecture the following: the filling radius of the complex projective space $C P^{k}$ with sectional curvature between ${ }_{4}^{1}$ and 1 equals half the spherical diameter of the $(2 k+2)$-point set of Proposition 1 .

The filling radius of a Riemannian manifold $X$ is defined as the infimum of all $\varepsilon>0$ such that $X$, isometrically imbedded in some other space, bounds in its $\varepsilon$-neighborhood $U_{\varepsilon} X$; i.e., $i_{F}[X]=0$ where $[X]$ is the fundamental homology class of $X$ and $i_{k}$ is induced by the imbedding $X \hookrightarrow U_{*} X$.

A construction in the Banach space $L^{x}\left(C P^{m}\right)$ shows that the filling radius is no greater than the number given above. Missing from the proof of the opposite inequality is geometrical control of minimal subsets of diameter $<2 \pi / 3$ of $C P^{m}$.

B. Katz of the AI Lab of MIT should have been a co-author of this paper, were it not for his self-effacing modesty. I thank him sincerely for dedication and reliability prevalent in his programming and computing work to obtain new examples of minimal sets. These examples are the sole justification for the material of Sections 2, 4, and 7. My thanks are also due to the University Paris 6 for hospitality during the academic years 1981-1983 when I obtained the results of Sections 1 and 3, and to R. Spatzier for some subtle suggestions.

\section{The Digon Construction of Minimal Sets}

We define a digon to be the convex region on the sphere bounded by two meridians (great semicircles joining north and south poles). Let $\beta<\pi / 2$ be the angle of the digon and let $P$ be a point on one of the two meridians. If $\beta<r<$ distance from $P$ to the nearest pole\}, then the circle of radius $r$ centered at $P$ intersects the other meridian in two distinct points. Our preliminary construction is as follows.

We start at the north pole and "walk" south with step $r$ on alternating meridians.

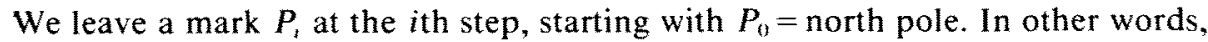
we proceed to partition the digon into isosceles triangles $\Delta_{r}$ with side $r$, starting with $\Delta_{1}$ which has two angles equal to $\beta$ (Fig. 1). Note that the even-numbered marks $P_{0}, P_{2}, \ldots$ all lie on one meridian.

Let $d_{i}=\operatorname{dist}\left(P_{0}, P_{1}\right)$. Then the increasing sequence $\left\{d_{i}\right\}$ satisfies the recurrence relation $\cos r=\cos d_{1} \cos d_{1+1}+\sin d_{1} \sin d_{t+1} \cos \beta$ (theorem of $\operatorname{cosines~[4,~p.~25]).~}$

If step $r$ equals $\beta$, it is clear that infinitely many steps will be required to reach the equator. As the construction depends continuously on $r$, we may choose values of $r>\beta$ so that the last mark $P_{k}$ north of the equator and the first mark $P_{k+1}$ south of it are equidistant from the equator, i.e., $d_{k}+d_{k+1}=\pi$. Then $P_{2 k+1}=$ south pole, and the resulting partition of the digon is invariant under the central symmetry around its center.

1.1. Proof of Proposition 1. The sets with $2 k+2$ points are just pyramids over $(2 k+1)$-gons (see the Introduction). They have rotational symmetry of order $2 k+1$. The sets with $3 k+1$ points have rotational symmetry of order 3 . To 


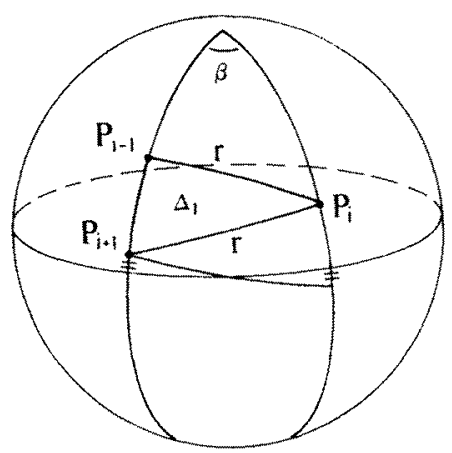

Fig. 1

construct them, we let $\beta=\pi / 3$. Consider the action of $Z_{3}$ on $S^{2}$ by rotations by multiples of $2 \pi / 3$ around $P_{0}=$ north pole. Then the orbit of the set $\left\{P_{0}, P_{2}, \ldots, P_{2 k}\right\}$ under the action of $Z_{3}$ is a pointwise extremal subset of $S^{2}$ with $3 k+1$ points. Its diameter $d=d_{2 k}$ satisfies $d+r=\pi$. Note that the orbit of $P_{k}$ (or $P_{k+1}$ if $k$ is odd) is an equilateral triangle with side $d$.

We will see in Section 6 that these sets are in fact minimal.

1.2. Remark. The limiting configuration of the $(2 k+2)$ family is the set consisting of the north pole and the circle at latitude $-\pi / 6$. The limiting configuration of the $(3 k+1)$ family is a countable set with three accumulation points on the equator.

It would be interesting to study the limiting configurations of general pointwise extremal sets of diameter $<2 \pi / 3$.

Let $\beta=\arccos 1 / n$. Consider the $n+1$ meridians passing through the vertices of a regular $n$-simplex inscribed in the equator $S^{n-1} \subset S^{n}$. On each meridian, take the point at spherical distance $d_{i}$ from the north pole, $i=2,4, \ldots, 2 k$. These points, together with the north pole, define a pointwise extremal subset of $S^{n}$ with $n k+k+1$ points.

A similar construction was independently described by Lovász [11].

When $n=1$ the above construction yields the $(2 k+1)$-gons in $S^{1}$.

\section{Separability and the Justification of Computer-Generated Sets}

We show how to construct new pointwise extremal sets starting with those of Section 1 , by means of an iterative computer procedure. This procedure relies on a property that may be called "separability."

2.1. Definition. Let $Y \subset S^{2}$, and suppose diam $Y<2 \pi / 3$. We say that points $x, y \in Y$ are separated by $Y$ if there are points $z, w \in Y$ such that the $\operatorname{set}\{x, y, z, w\}$ does not lie in any hemisphere. $Y$ is called separable if every pair of points of $Y$ are separated by $Y$. 
A pointwise extremal set is necessarily separable (see 4.1 ). Thus all sets of Proposition 1 are separable. Moreover, the sets of the $(3 k+1)$ family have many separable subsets.

If $\hat{Y} \in D\left(S^{2}, 2 \pi / 3\right)$, then the unique minimal set $Y$ in the connected component of $\hat{Y}$ is pointwise extremal (cf. Corollary 2 of the Introduction and Corollary 3.4 of Section 3).

2.2. Lemma. Suppose $\hat{Y}$ is separable. Then Yand $\hat{Y}$ have the same number of points.

Proof. Three points on $S^{2}$ define a convex spherical triangle. Four points on $S^{2}$ define four triangles whose sum is a 2-cycle. If the 4-tuple does not lie in any hemisphere, this 2-cycle belongs to the generator of $\mathrm{H}_{2}\left(\mathrm{~S}^{2}, Z_{2}\right)$. The lemma follows from the fact that if all pairwise distances are $<2 \pi / 3$, then the 2 -cycle depends continuously on the four points and hence the homology class is fixed.

2.3. Question. We have defined a map $\varphi: Y^{4} \rightarrow Z_{2}$ which associates to each 4-tuple the corresponding element of $\mathrm{H}_{2}\left(S^{2}, Z_{2}\right)$. Does $\varphi$ determine the minimal set $Y \subset S^{2}$ up to congruence?

To construct a new minimal set $Y$, we choose a separable subset $\hat{Y}$ of a $(3 k+1)$-point set of Proposition 1. Let $n=\# \hat{Y}$. We replace $D\left(S^{2}, 2 \pi / 3\right)$ by a finite-dimensional configuration space $V$ defined as the $n$-fold Cartesian product of copies of $S^{2}$. We may view $\hat{Y}$ as a point of $V$.

At every point of $V$, consider the "gradient" vector pointing in the direction of greatest decrease of the diameter function on $V$, and of length equal to the partial derivative in that direction. We move $\hat{Y}$ along this vector field to a zero of the gradient field. Thus we obtain a critical point of the diameter function which is a minimal set by Theorem 2 . A recursive simulation of this procedure can be done by computer. The recursion produces a set which is approximately minimal, and Proposition 7.1 tells us when there is a genuine minimal set nearby.

If we apply the recursive procedure which moves only one point of $\hat{Y}$ at a time, then we may get a set which is approximately pointwise extremal but not approximately minimal, in a sense explained in Section 7. In that case Proposition

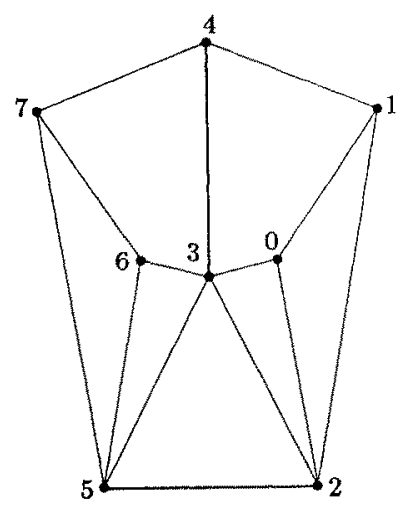

Fig. 2 
Table 1

\begin{tabular}{cll}
\hline Point & \multicolumn{1}{c}{ Source } & \multicolumn{1}{c}{ Coordinates } \\
\hline 0 & North pole & $(0.596,0.207)$ \\
1 & $R^{2 \pi / 3} P_{2}$ & $(-0.616,1.076)$ \\
2 & $R^{4 \pi / 3} P_{2}$ & $(-0.616,-1.076)$ \\
3 & $P_{4}$ & $(1.648,0.0)$ \\
4 & $R^{2 \pi / 3} P_{4}$ & $(-0.816,1.411)$ \\
5 & $R^{4 \pi / 3} P_{4}$ & $(-0.816,-1.411)$ \\
6 & $P_{6}$ & $(2.702,0.0)$ \\
7 & $R^{2 \pi / 3} P_{6}$ & $(-1.17,1.632)$ \\
\hline
\end{tabular}

7.1 is not applicable, and extra work would have to be done to show that there is a pointwise extremal set nearby.

To give an example, we consider the set with $3 k+1$ points of Proposition 1 for $k=3$ (see 1.1). Remove the points $P_{2}$ and $R^{4 \pi / 3} P_{6}$. The computer then produces the set of Fig. 2, with the points labeled as in Table 1; the latter also contains the exponential coordinates to three decimals. The graph in the figure is defined by the tiling (cf. Section 3 ).

\section{The Structure of Pointwise Extremal Sets}

In this section we prove Theorem 1 and derive further properties of pointwise extremal sets.

Suppose $Y$ is as in Theorem 1. Let $y \in Y$. Let $H_{y} \subset S^{2}$ denote the spherial convex hull of the set $\{x \in Y \mid \operatorname{dist}(x, y)=d\}$. Since $d<2 \pi / 3, H_{y}$ is a nondegenerate spherical polygon. The polygon $H_{y}$ is inscribed in the circle of radius $\pi-d$ centered at $y^{\prime}=$ the point of $S^{2}$ opposite $y$. The vertices of $H_{y}$ are precisely the points of $Y$ at distance $d$ from $y$.

3.1. Remarks. 1. For every vertex $x \in H_{y}$, the vector $x^{\prime}$ points inside $H_{y}$. This is because, by assumption, $y$ is held by $Y$.

2. No point of $Y$ can lie on a side of $H_{y}$.

Two adjacent vertices $a, b$ of $H_{y}$ determine a convex cone $a y^{\prime} b$ of directions at $y^{\prime}$, leading to no other vertices of $H_{y}$. Such a cone is called a sector at $y^{\prime}$. In the antipodal picture, points $a^{\prime}$ and $b^{\prime}$ define a cosector, i.e., a cone of directions at $y$.

3.2. Lemma. Suppose $Y \subset S^{2}$ is pointwise extremal with diam $Y=d<2 \pi / 3$. Then the sphere is tiled by the polygons $H_{y}$ :

$$
\bigcup_{y \in Y} H_{y}=S^{2}, \quad \stackrel{\circ}{H}_{y} \cap \stackrel{\circ}{H}_{x}=\varnothing \quad \text { if } y \neq x .
$$

Proof. If $\stackrel{\circ}{H}_{y} \cap \stackrel{\circ}{H}_{x}$ is nonempty then some vertex $z$ of $H_{x}$ lies in $\stackrel{\circ}{H}_{y}$ (or vice versa) and so $d \geq \operatorname{dist}(y, z)>\operatorname{dist}\left(y, H_{y}\right)=d$. 


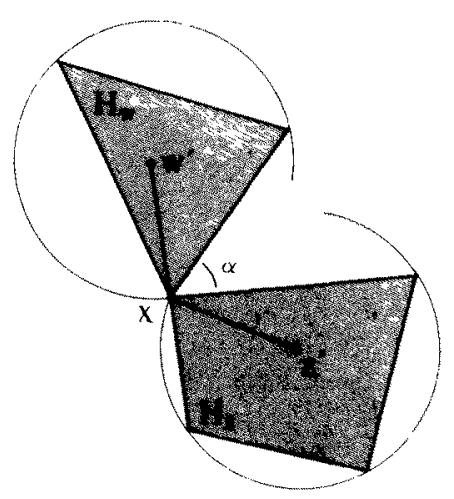

Fig. 3

Suppose $C$ is a connected component of the complement $S^{2} \backslash \bigcup_{1}, H_{3}$. Then $C$ has convex boundary $\partial C$. Indeed, let us show that any interior angle $\alpha$ of $C$, with vertex $x$, is acute. Let $H_{n}$ and $H$. be the polygons adjacent to $\alpha$ (Fig. 3). Then $x$ is a vertex of both $H_{*}$ and $H_{z}$ by Remark 3.1(2). Every tangent vector in the cone $n^{\prime} x z^{\prime}$ facing $C$ is contained in either $H_{n}, C$, or $H_{z}$. Hence by Remark $3.1(1), w^{\prime} x z^{\prime}$ is a cosector. Thus $\alpha$ lies in a cosector and is acute.

It follows that $\partial C$ is simple. To see this, suppose we approach the boundary of $C$ in two disjoint neighborhoods in its interior. Consider the shortest curve in $C$ joining the two boundary points. Because $\partial C$ is convex, this curve lies in the interior of $C$ and therefore is a minimizing arc of great circle. Hence the two boundary points are distinct as points on $S^{2}$.

Similarly, one can show that $C$ is simply connected and hence $\partial C$ is connected. We view $\partial C$ as a polygonal curve and label its vertices $a_{1}, \ldots, a_{m}(m \geq 3)$ so that the side $a_{t} a_{t+1} \subset H_{t}=H_{1}$, (Fig. 4) (the indices are $\bmod m$ ). Consider the simple closed polygonal curve $E=a_{1} y_{1}^{\prime} a_{2} y_{2}^{\prime} \cdots a_{m} y_{m}^{\prime}$. The angles of $E$ facing $C$ are alternating sectors and cosectors. Hence $E$ is a convex curve. It is easy to

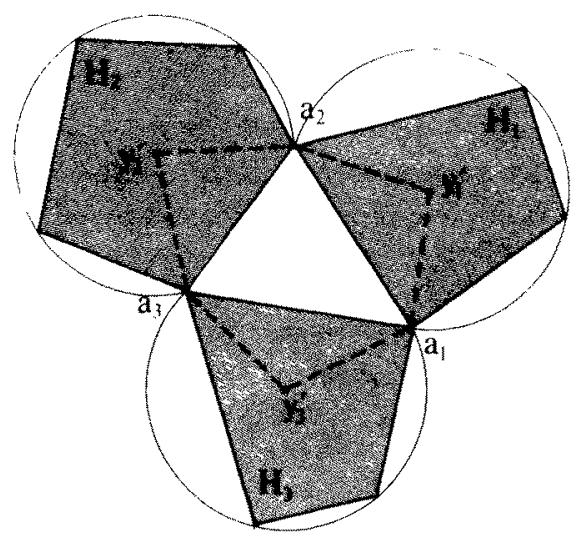

Fig. 4 
see that a convex simple closed curve on $S^{2}$ has length at most $2 \pi$ (with equality only for a digon described in Section 1). On the other hand, length $E=2 m r \geq 6 r>$ $2 \pi$, where $r=\pi-d$. The contradiction shows that the $H_{v}$ tile is $S^{2}$.

3.3. Corollary. Suppose $Y \subset S^{2}$ is pointwise extremal with diam $Y=d<2 \pi / 3$. Then:

1. For every point $p \in S^{2}$, there is a point $y \in Y$ with $\operatorname{dist}(p, y) \geq d$, with equality (for the farthest $y$ ) if and only if $p \in Y$.

2. No proper subset $Y^{\prime} \subset Y$ with diam $Y^{\prime}=d$ is pointwise extremal.

The proof is obvious.

In particular, the graph whose vertices are points of $Y$ and whose edges correspond to pairs of points of $Y$ at distance $d$, is connected. Corollary 6.3 will provide a more detailed description of this graph.

3.4. Corollary. Suppose $Y \in D\left(S^{2}, 2 \pi / 3\right)$ is a critical point of the diameter function. Then $Y$ is pointwise extremal.

Proof. Note that without the diameter restriction $Y$ may not be pointwise extremal, e.g., the 4-point set $Y_{4}$ consisting of the north pole and the vertices of an equilateral triangle inscribed in the equator (of course, diam $Y_{4}=2 \pi / 3$ so $\left.Y_{4} \notin D\left(S^{2}, 2 \pi / 3\right)\right)$.

Let $d=\operatorname{diam} Y$. Let $Y_{0} \subset Y$ be defined by $Y_{0}=\left\{y \in Y \mid \operatorname{diam}_{y} Y=d\right\}$, where $\operatorname{diam}_{x} Y \equiv \max _{z \in Y} \operatorname{dist}(x, z)$. The set $Y_{0} \in D\left(S^{2}, 2 \pi / 3\right)$ is a critical point of the diameter function but may not be pointwise extremal. Note that if $y \in Y_{0}$ is not held by $Y_{0}$ (see the Introduction) then we can perturb $y$ so as to decrease $\operatorname{diam}_{y} Y_{0}$ linearly in the size of the perturbation.

We define the sets $Y_{i}$ recursively by

$$
Y_{i}=\left\{y \in Y_{i-1} \mid y \text { is held by } Y_{i-1}\right\}, \quad i=1,2, \ldots
$$

The sequence must stabilize before we exhaust $Y_{0}$, for otherwise we could construct a perturbation linearly decreasing diam $Y_{0}$, contradicting the hypothesis. The stable set $Y^{\prime} \subset Y$ is both pointwise extremal and a critical point of the diameter function, and hence $Y^{\prime}=Y$ by Corollary 3.3(1).

Proof of Theorem 1. The spherical graph defined by the tiling has $n$ vertices and $n$ faces. Hence the graph has $2 n-2$ edges by Euler's theorem [4, p. 15]. A vertex $v$ with valence $k$ lies opposite a $k$-gonal face $f$. Let $e$ be the number of edges and $m$ the number of pairs of points at distance $d$. Then $2 e=\sum k(v)$ while $2 m=\sum k(f)$. Therefore $m=e=2 n-2$. The theorem is proved.

\section{Approximately Extremal Sets}

We prove an analog of Theorem 1 for sets which are almost pointwise extremal. This analog (Proposition 4.6) is proved under the hypothesis that sufficiently 


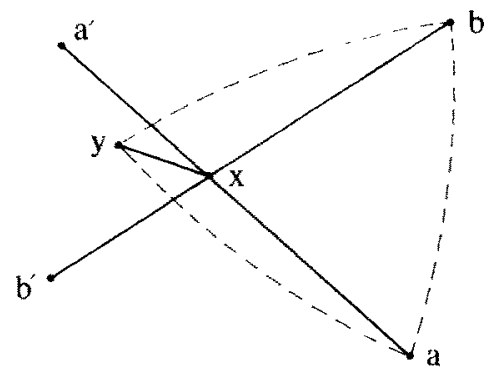

Fig. 5

many of the pairwise distances are approximately equal, and that in addition the set is separable in the sense of 2.1.

4.1. Lemma. Suppose $Y \subset S^{2}$ is pointwise extremal with diam $Y=d<2 \pi / 3$. Then $Y$ is separable.

Proof. Let $x, y \in Y$. Let $a^{\prime} x b^{\prime}$, where $a, b \in Y$, be the cosector (see 3.1) containing the vector $\mathbf{x y}$. If the set $\{x, y, a, b\}$ is contained in some hemisphere, then $x$ is contained in the (convex) triangle bay (Fig. 5). Therefore $b y+a y>b x+a x=2 d$, and hence either $b y>d$ or $a y>d$. The contradiction proves the lemma.

4.2. Corollary. Let $Y \in D\left(S^{2}, 2 \pi / 3\right)$. Let $x, y \in Y$. Define a relation $\sim$ among the points of $Y$ by

$$
x \sim y \Leftrightarrow \varphi(x, y, z, w)=0 \quad \text { for all } z, w \in Y
$$

(cf. 2.3). Then $\sim$ is an equivalence relation.

Proof. Clearly, $x-y$ if and only if $x$ and $y$ collapse to the same point of any pointwise extremal set in the connected component of $Y \in D\left(S^{2}, 2 \pi / 3\right)$ (cf. 2.1).

4.3. Lemma. Suppose points $P_{i} \in S^{2}, i=1,2,3,4$, do not lie in any hemisphere. Let $\pi / 2 \leq d<2 \pi / 3$, and assume $\operatorname{dist}\left(P_{i}, P_{j}\right) \leq d$ for all $i, j$. Then

$$
\cos \operatorname{dist}\left(P_{1}, P_{2}\right) \leq 2 \frac{\cos ^{2} d}{\cos ^{2}(d / 2)}-1,
$$

with equality if and only if triangles $P_{1} P_{3} P_{4}$ and $P_{2} P_{3} P_{4}$ are equilateral with sided.

To prove Lemma 4.3 we will need the lemma below which will also be useful in Section 7 (see 6.6).

4.4. Definition. Denote by $\alpha(d)$ the angle of the equilateral spherical triangle with side $d$. Then $2 \cos (d / 2) \sin (\alpha(d) / 2)=1$. 
4.5. Lemma. Let $A B C$ be a spherical triangle with sides of length $\leq d$, and assume that sides $A B$ and $A C$ are $\geq \pi / 2$. Then $\angle B A C \leq \alpha(d)$.

Proof. By the theorem of cosines

$$
\cos \angle B A C=\frac{\cos B C-\cos A B \cos A C}{\sin A B \sin A C} \geq \frac{\cos d}{\sin A B \sin A C}-\cot A B \cot A C .
$$

Since $\cos d \leq 0$, we have

$$
\frac{\cos d}{\sin A B \sin A C} \geq \frac{\cos d}{\sin ^{2} d}
$$

and since $A B, A C \geq \pi / 2$, we have $\cot A B \cot A C \leq \cot ^{2} d$. Hence $\cos \angle B A C \geq$ $(\cos d) /\left(\sin ^{2} d\right)-\cot ^{2} d=\cos \alpha(d)$.

Proof of Lemma 4.3. Denote by $U$ the point of intersection of the shortest arc $P_{3} P_{4}$ and the great circle through $P_{1}$ and $P_{2}$, so that $U P_{1}+P_{1} P_{2}+P_{2} U=2 \pi$. To prove the lemma it suffices to show that $U P_{1}$ is less than the height of the equilateral spherical triangle with side $d$. By first variation, $U P_{1} \leq$ $\max \left(P_{4} P_{1}, P_{3} P_{1}, h\right)$, where $h$ is the length of the perpendicular dropped from $P_{1}$ to (possibly the continuation of) $P_{3} P_{4}$. We may assume that $h \geq P_{3} P_{1}$; then the angle $\angle P_{1} P_{3} P_{4}$ is obtuse. Spherical trigonometry gives

$$
\sin h=\sin P_{1} P_{3} \sin \left(\angle P_{1} P_{3} P_{4}\right) \geq \sin d \sin \alpha(d)
$$

by Lemma 4.5 . The lemma is proved.

Together, Lemmas 4.1 and 4.3 show that the distance between two points of a pointwise extremal set can be bounded from below in terms of the diameter of the set. Thus the number of points $n$ in such a set can be bounded from above in terms of $d$ by a volume argument. It is not clear if the converse is true: can $d$ be bounded away from $2 \pi / 3$ in terms of $n$ ?

Let $C$ be a collection of pairs of points of a set $Y \subset S^{2}$. In analogy with the definition given in the Introduction, we say that a point $y \in Y$ is held by $C$ if, for every tangent vector $v \in T_{y} S^{2}$, there is a pair $\{x, y\} \in C$ such that $v \cdot \mathbf{y x} \geq 0$. By abuse of notation, we will not distinguish between a pair of points and their distance.

4.6. Proposition. Let $Y \subset S^{2}$, \#Y=n, diam $Y=d=2 \pi / 3-t$, where $t>0$. Let $d_{1} \geq \cdots \geq d_{m}$ be the $m$ largest distances among points of $Y$. Suppose the following three conditions are satisfied:

1. $Y$ is separable (cf. 2.1).

2. Each point of $Y$ is held by the collection $\left\{d_{k}\right\}, k=1, \ldots, m$.

3. $d_{1}-d_{m}<t$.

Then $m=2 n-2$.

4.7. Corollary. If $d_{1}-d_{m}<t / 2$ then $d_{m}-d_{m+1}>t / 2$, i.e., the $(2 n-1)$ th largest distance $d_{2 n-1}$ is "much" smaller than $d_{2 n-2}$. 
Proof of Proposition 4.6. Let $y \in Y$. To define an analog of $H_{3}$ we consider the vectors $\mathbf{y x}$, where $\left\{x_{i}, y\right\} \in\left\{d_{k}\right\}$, ordered clockwise, and construct a polygon $H_{4}$ by joining the consecutive points $x_{r}$ by shortest arcs. Note that $\ddot{H}_{z} \cap \mathscr{\mathscr { H }}_{z}=\varnothing$ if $y \neq z$, for otherwise a vertex $w$ of $H_{z}$ must lie in $H_{3}$ (or vice versa) and hence $\operatorname{dist}(y, w)>\operatorname{dist}\left(y, H_{\mathrm{y}}\right) \geq d_{m}$, so that $w$ must must be a vertex of $H_{v}$. We will show that the polygon $H_{v}$, while no longer inscribed in a circle, is convex. Then the proposition follows from an analog of Lemma 3.2 by the argument of the proof of Theorem 1.

To see that $H_{v}$ is convex, it suffices to show that each of the angles $\angle y^{\prime} x_{r} x_{1+1}$ and $\angle y^{\prime} x_{1} x_{1-1}$ is acute. Let $x_{1}=a$ and $x_{r+1}\left(\right.$ or $\left.x_{1-1}\right)=b$. We have

$$
\cos \angle y^{\prime} a b=\frac{\cos y^{\prime} b-\cos y^{\prime} a \cos a b}{\sin y^{\prime} a \sin a b} .
$$

To show that this is positive, we estimate the numerator using Lemma 4.3:

$$
\begin{aligned}
\cos y^{\prime} b-\cos y^{\prime} a \cos a b & =\cos y a \cos a b-\cos y b \\
& \geq \cos d_{1} \cos a b-\cos d_{m} \\
& =\cos d_{1}(\cos a b-1)-\left(\cos d_{m}-\cos d_{1}\right) \\
& \geq \cos d_{1}(\cos a b-1)-\left(d_{1}-d_{m}\right) \\
& \geq \cos d\left(2 \frac{\cos ^{2} d}{\cos ^{2}(d / 2)}-2\right)-t
\end{aligned}
$$

Since $\arccos \left(-\frac{1}{3}\right) \leq d<2 \pi / 3$ and $\arccos (1 / \sqrt{3}) \leq d / 2<\pi / 3$ (see the Remark following Proposition 1 of the Introduction), we have

$$
\begin{aligned}
\cos d\left(2 \frac{\cos ^{2} d}{\cos ^{2}(d / 2)}-2\right) & =2(-\cos d) \frac{1}{\cos ^{2}(d / 2)}\left(\cos \frac{d}{2}-\cos d\right)\left(\cos \frac{d}{2}+\cos d\right) \\
& \geq 2 \cdot\left(\frac{1}{3}\right) \cdot(3) \cdot\left(\frac{1}{2}\right) \cdot\left(\cos \frac{d}{2}+\cos d\right) \\
& =\cos \left(\frac{\pi}{3}-\frac{t}{2}\right)+\cos \left(\frac{2 \pi}{3}-t\right) \\
& =\frac{1}{2} \cos \frac{t}{2}+\frac{\sqrt{3}}{2} \sin \frac{t}{2}-\frac{1}{2} \cos t+\frac{\sqrt{3}}{2} \sin t \\
& \geq \frac{\sqrt{3}}{2}\left(\sin \frac{t}{2}+\sin t\right) \\
& =\frac{\sqrt{3}}{2} \int_{0}^{t}\left(\frac{1}{2} \cos \frac{t}{2}+\cos t\right) d t \\
& \geq \frac{\sqrt{3}}{2} \int_{0}^{t} \frac{3}{2} \cos t d t
\end{aligned}
$$




$$
\begin{aligned}
& \geq \frac{3 \sqrt{3}}{4} \cdot \frac{\sqrt{3}}{2} t \quad\left(\text { as } t<\frac{2 \pi}{3}-\frac{\pi}{2}=\frac{\pi}{6}\right) \\
& <t .
\end{aligned}
$$

Thus $H_{v}$ is convex and the proposition is proved.

\section{Walkup's Inequality and Minimal Subsets of $S^{3}$}

Let $Y \subset S^{3}$ and assume diam $Y<\arccos \left(-\frac{1}{3}\right)$. Then a map $\varphi: Y^{5} \rightarrow H_{3}\left(S^{3}, Z_{2}\right)$ is defined similarly to 2.3 .

Let us carry out a heuristic count to guess a suitable analog to Theorem 1. Suppose $Y$ is pointwise extremal, $\operatorname{diam} Y=d, \# Y=n$. Each point of $Y$ contributes 3 degrees of freedom, but $\operatorname{dim} S O(4)=6$ and the actual value of $d$ is a parameter which decreases by 1 the effective number of constraints imposed by pairs of points at distance $d$. Thus one would expect to have $3 n-6+1=3 n-5$ pairs of points at distance $d$. (A similar argument for $S^{2}$ yields the correct number $2 n-\operatorname{dim} S O(3)+1=2 n-2$.) In fact, all the computer-generated examples so far had $3 n-5$ such pairs.

Borsuk's conjecture states that every set of diameter 1 in $R^{n}$ can be covered by $n+1$ sets of diameter $<1$. The conjecture has been verified for $R^{2}$ and $R^{3}$, but one suspects that it is false in general.

If one tried to prove Borsuk's conjecture for a set $Y \subset S^{3} \subset R^{4}$ as above by the method of [8], [9], and [16], the best one could hope for is to cover $Y$ by six sets of smaller diameter. This might be an indication that the constant in Borsuk's conjecture for $R^{\prime}$ ( $s \geq 3$ ) should be $2(s-1)$ instead of $s+1$ (when $s=2$, the count changes due to the cancellation of $\operatorname{dim} S O(2)$ and 1$)$.

5.1. We may define the spherical convex polyhedra $H_{v}$ in analogy with Section 3. Under the assumption that the $H_{1}$ tile $S^{3}$ (i.e., the Euclidean convex hull of $Y$ is a self-dual 4-polytope), we may carry out the following calculation.

First, assume $P$ is an arbitrary 4-polytope. We refer to 1-faces, 2-faces, and 3-faces of $P$ respectfully as edges, faces, and cells. For each cell $c$ of $P$ we have Euler's theorem $v_{c}-e_{i}+f_{i}=2$. Also $e_{c}=\frac{1}{2}\left(3 a_{c}^{3}+4 a_{c}^{4}+5 a_{i}^{5}+\cdots\right)=$ $\frac{3}{2} f_{c}+\frac{1}{2}\left(a_{\imath}^{4}+2 a_{q}^{5}+\cdots\right)$, where $a_{i}^{\prime}$ is the number of $j$-gonal faces of $c$. We have

$$
v_{\mathrm{k}}=2+\frac{1}{2} f_{\mathrm{c}}+\frac{1}{2}\left(a_{c}^{4}+2 a_{c}^{5}+\cdots\right) \text {. }
$$

Summing over all cells of $P$ we get

$$
\sum_{c} v_{c}=2 c+\frac{1}{2} \sum_{c} f_{c}+\sum_{2}^{1} \sum_{c}\left(a_{c}^{4}+2 a_{c}^{5}+\cdots\right)=2 c+f+\left(a_{4}+2 a_{5}+\cdots\right) \text {. }
$$

Here $c$ is the number of cells of $P, f$ is the number of faces of $P$, and $a$, is the number of $j$-gonal faces of $P$.

Now we apply the procedure of pulling the vertices of $P$ (see p. 116 of [13]) to construct a simplicial polytope $Q$ with the same number of vertices $v$ as $P$. 
Let $E$ and $e$ be the numbers of edges of $Q$ and $P$, respectively. The description of $Q$ given in [13] allows one to write down the following formula for $E$ :

$$
E=e+\left(a_{4}+2 a_{5}+\cdots\right)+\text { "cell diagonals," }
$$

where cell diagonals (c.d.) are the edges joining pairs of vertices of $Q$ which did not belong to any common face of $P$ (not all such pairs need be joined by an edge of $Q$ ). Therefore $\sum_{c} v_{c}=2 c+f+E-e-c$.d.

Walkup [17] proved that $E \geq 4 v-10$. (This is a special case of McMullen's conjecture proved by Stanley [15] and Billera and Lee [1].) Hence $\sum, v_{c} \geq$ $2 c+f-e+4 v-10-$ c.d.

Finally, if $P$ is as in 5.1, then $f=e$ and $v=c=n$. Also, $\sum_{c} v_{c}=2 N$, where $N$ is the number of pairs of points at maximal distance. Hence

$$
N \geq 3 n-5-\frac{1}{2} \text { c.d. }
$$

For example, the pyramid over the set with $3 k+1$ points for $k=3$ of Proposition 1 (see Section 1) has $n=11$ and $N=3 n-5=28$. But it also has the property that any nearby simplicial polytope must possess cell diagonals. Hence it is not extremal for Walkup's inequality.

\section{Caratheodory and the Proof of Theorem 2}

Let $D_{s}\left(S^{2}, d\right) \subset D\left(S^{2}, d\right)$ denote the space of separable subsets of $S^{2}$ (see 2.1 ). It follows from Corollary 4.2 that $Y \in D$ is a critical point of the diameter function on $D$ if and only if it is a critical point of its restriction to $D_{s}$.

On the other hand, $D_{s}$ locally imbeds in the $n$-fold Cartesian product $V=$ $S^{2} \times \cdots \times S^{2}$, with typical point $Y=\left(y_{1}, \ldots, y_{n}\right)$. Denote by $\pi_{t}: V \rightarrow S^{2}$ the projection to the $i$ th factor. Consider the function $f_{y}$ on $V$ defined by $f_{i j}\left(Y^{\prime}\right)=$ $\operatorname{dist}\left(\pi_{i}(Y), \pi_{j}(Y)\right)$. Clearly,

$$
T_{Y} \pi_{i}\left(\nabla f_{i j}\right)=\mathbf{y}_{i} \mathbf{y}_{j} \quad \text { and } \quad T_{Y} \pi_{i}\left(\nabla f_{m j}\right)=0 \quad \text { if } m \neq i .
$$

Here $\mathbf{y z} \in T_{y} S^{2}$ denotes the unit tangent vector to the shortest arc joining $y$ with $z$.

If $Y \subset S^{2}$ is pointwise extremal with diam $Y=d$, then there are $2 n-2$ pairs $(i, j)$ such that $f_{i j}(Y)=d$ by Theorem 1 . We relabel these functions by $f_{k}$, $k=1, \ldots, 2 n-2$.

The orthogonal group $S O(3)$ acts on $V$ by $g \cdot Y=\left(g y_{1}, \ldots, g y_{n}\right)$. The orbit of $Y$ under this action is three-dimensional as long as $Y$ contains a pair of distinct nonantipodal points. Let $N=N_{Y} \subset T_{Y} V$ be the tangent space to the orbit.

The $d f_{k} \in T^{*} V$ satisfy $d f_{k}(N) \equiv 0$, and hence span at most a $(2 n-$ 3)-dimensional subspace of $T^{*} V$. Thus they are linearly dependent:

$$
\sum_{k=1}^{2 n-2} a_{k} d f_{k}=0
$$


Let $a\left(y_{1}, y_{1}\right)$ be the coefficient of $f_{i f}$ in this sum. By $(*)$, equation (**) is equivalent to the following system of equations:

$$
\sum_{h, H,} a(h, y) \mathbf{y h}=0 \quad \text { for every } y \in Y
$$

where the summation is taken over all vertices of $H_{1}$ (see Section 3 ).

6.1. Lemma. Let $Y$ be a pointwise extremal set. Suppose the coefficients in $(* *)$ satisfy $a_{k} \geq 0$ for all $k$. Then $a_{k}>0$ for all $k$.

We will need the following extension of the results of Section 3 to prove Lemma 6.1.

6.2. Lemma. Let $Y \subset S^{2}$ be a pointwise extremal set with diam $Y=d<2 \pi / 3$. Let $x, y \in Y$ with $\operatorname{dist}(x, y)=d$. Then either $x$ is not held by $Y \backslash\{y\}$ or $y$ is not held by $Y \backslash\{x\}$.

Proof. Let $a$ and $b$ be the vertices of the face $H$, which are adjacent to $x$. If $y$ is held by $Y \backslash\{x\}$ then the union of the sectors $a y^{\prime} x$ and $x y^{\prime} b$ is still a convex cone at $y^{\prime}$. By Lemma 3.2 there is a face $H_{z}$ bordering $H_{1}$ on the side $a x$, and a face $H_{w}$ bordering $H_{1}$, on $x b$ (Fig. 6). If $x$ is held by $Y \backslash\{y\}$ then the union of the cosectors $z^{\prime} x y^{\prime}$ and $y^{\prime} x w^{\prime}$ is a convex cone. Thus the sum of the four angles involved is $<2 \pi$.

On the other hand, the angles of the "diamond" $x z^{\prime} a y^{\prime}$ satisfy $\angle z^{\prime} x y^{\prime}+\angle x y^{\prime} a>$ $\pi$, and, similarly, $\angle w^{\prime} x y^{\prime}+\angle x y^{\prime} b>\pi$. The contradiction shows that each pair of points at distance $d$ is indispensable for the pointwise extremality of $Y$. We are grateful to $B$. Heepe for pointing out that the diameter restriction should not play an essential role in the proof.

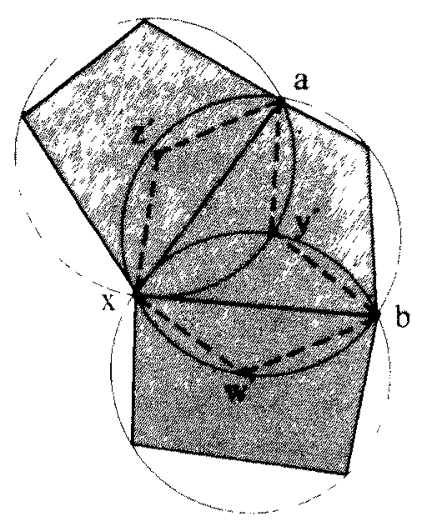

Fig. 6 
6.3. Corollary. Let $\left\{x_{i}, y_{i}\right\}, i=1,2$ with $\operatorname{dist}\left(x_{i}, y_{i}\right)=d$, be unordered pairs of points of $Y$. Then there is a sequence of ordered pairs $p_{k}, k=1, \ldots, m$, such that the following four properties are satisfied:

1. $p_{1}$ is an ordering of $\left\{x_{1}, y_{1}\right\}$ and $p_{m}$ is an ordering of $\left\{x_{2}, y_{2}\right\}$.

2. $\operatorname{end}\left(p_{k}\right)=\operatorname{init}\left(p_{k+1}\right), k=1, \ldots, m-1$, where end $(x, y)=y$ and $\operatorname{init}(x, y)=x$.

3. end $\left(p_{k}\right)$ is not held by $Y \backslash$ init $\left.\left(p_{k}\right)\right\}, k=1, \ldots, m-1$.

4. $m \leq n=$ the number of points of $Y$.

Proof. This follows from Lemma 6.2 and Corollary 3.3(2).

Proof of Lemma 6.1. Since $(* *)$ is equivalent to $(* * *)$, it suffices to show that if $a(x, y)=0$ for some $x, y \in Y$, then all the coefficients must vanish.

If $y$ is not held by $Y \backslash\{x\}$, then the vectors yh, $h \in H_{y} \backslash\{x\}$ all lie in an open halfspace. Since all $a_{k} \geq 0,(* * *)$ implies $a(h, y)=0$ for all $h \in H_{v}$. Now the lemma follows from Corollary 6.3 .

Proof of Theorem 2. If $Y \in D\left(S^{2}, 2 \pi / 3\right)$ is a critical point of the diameter function then by a first variation argument there are coefficients $a_{k} \geq 0$ such that $\sum a_{k} d f_{h}=0$ at the point $Y \in V$. (Recail that $V=S^{2} \times \cdots \times S^{2}$, and $N \subset T_{Y} V$ is the tangent space to the $S O(3)$-orbit.) Equivalently, for every $v \in T_{Y} V$ there is an $f_{k}$ such that $d f_{k}(v) \geq 0$. To show that $Y$ is a minimal set it suffices to prove that, for every $v \in T_{Y} V \backslash N$, there is a function $f_{k}$ with $d f_{k}(v)>0$. Suppose $d f_{k}(v) \leq 0$ for all $k$. By projecting to the line spanned by $v$ we see that, for every $k$, either $d f_{k}(v)=0$ or $a_{k}=0$. By Caratheodory's theorem (see p. 26 of [13]) there are coefficients $b_{k} \geq 0$ such that $\sum_{k=1}^{2 n-2} b_{k} d f_{k}=0$, where one of the coefficients $b_{k}$ equals 0 . But this contradicts Lemma 6.1. The theorem is proved.

Proof of Corollaries 1 and 2 of the Introduction. Corollary 1 is immediate from the compactness of $V$.

Suppose two minimal sets lie in the same connected component of $D_{s}\left(S^{2}, 2 \pi / 3\right)$. Then they also lie in the same connected component $A$ of $D_{\mathrm{s}}\left(S^{2}, d\right)$ for some $d<2 \pi / 3$. By Corollary 1 , we can choose a minimal set $Y \in A$ with the biggest diameter. Let $Z \in A$ be another minimal set. Let

$$
a=\inf \left\{c<d \mid Y \text { and } Z \text { lie in the same connected component of } D_{5}\left(S^{2}, c\right)\right\} .
$$

We have $a>\operatorname{diam} Y$ because $Y$ is a minimal set. Let $\varepsilon=\frac{1}{2} \min (d-a, a-\operatorname{diam} Y)$. Let $\gamma$ be a curve in $D_{s}\left(S^{2}, a+\varepsilon\right)$ joining $Y$ and $Z$ and transverse to the $S O(3)$ action.

There are no critical values in the interval $[a-\varepsilon, a+\varepsilon]$. We construct a smooth nonvanishing vector field which forms an acute angle with the "gradient" of the diameter function (cf. Section 2). This is done by patching together radially parallel fields supported in small balls as in the proof of the Isotopy lemma of [7] and [5, p. 182] for the distance function. We can push the curve $\gamma$ into $D_{s}\left(S^{2}, a-\varepsilon\right)$ by following this field, contrary to the definition of $a$. This proof of Corollary 2 is spiritually akin to the mountain pass lemma of Morse theory (see Lemma $4.11(2)$ of [3]). 
Remark. All the pointwise extremal sets of Proposition 1 are minimal subsets of $S^{2}$. To see this we must show that all the coefficients in $(* *)$ have the same sign. In the case of the sets of Proposition 1, this follows easily from the following two observations:

1. If $H_{v}$ is a triangle then all three coefficients $a(h, y), h \in H_{v}$, have the same sign.

2. Suppose $H_{y}$ is a quadrilateral with vertices $h_{t}, i=1, \ldots, 4$, labeled so that $y$ is held by $\left\{h_{1}, h_{2}, h_{3}\right\}$ and by $\left\{h_{1}, h_{2}, h_{4}\right\}$, but not by the remaining two triples. Suppose that $\operatorname{sign} a\left(h_{3}, y\right)=\operatorname{sign} a\left(h_{4}, y\right)$. Then all four signs are the same.

In Section 7 we will need the following two estimates. Let $\alpha(d)$ be defined as in 4.4.

6.4. Lemma. Let $Y \subset S^{2}$ be a minimal set, and let $n=\# Y, d=\operatorname{diam} Y<2 \pi / 3$. Then the coefficients of $(* *)$ satisfy

$$
\frac{\min _{k} a_{k}}{\max _{k} a_{k}} \geq\left(\cos \frac{\alpha(d)}{2}\right)^{n-1} .
$$

Proof. Let $y \in Y$. Assume $y$ is not held by $Y \backslash\{x\}$, and let $z$ and $w$ be the vertices of $H_{y}$ adjacent to $x$. Let $v \in T_{,} S^{2}$ be the bisector of $\angle z y w$. Then for every $h \in H_{v} \backslash\{x\}$, the vectors $v$ and yh define an angle $\leq \alpha(d) / 2$ at $y$, because $\operatorname{dist}(z, w) \leq d$. Let $\pi$ be the orthogonal projection of $T_{z} S^{2}$ to the line spanned by $v$. Then $\|\pi(\mathbf{y h})\| \geq$ $\cos (\alpha(d) / 2)$. Suppose for some $h_{0} \in H_{y}$ we have $a\left(h_{0}, y\right) \cos (\alpha(d) / 2)>a(x, y)$, where the coefficients are as in $(* * *)$. Then

$$
\begin{aligned}
0=\left\|\sum_{h} a(h, y) \mathbf{y h}\right\| & \geq\left\|\pi\left(\sum_{h} a(h, y) \mathbf{y h}\right)\right\| \\
& \geq\left\|a\left(h_{0}, y\right) \pi\left(\mathbf{y h} \mathbf{h}_{0}\right)+a(x, y) \pi(\mathbf{y x})\right\| \\
& \geq a\left(h_{0}, y\right) \cos \frac{\alpha(d)}{2}-a(x, y) \\
& >0 .
\end{aligned}
$$

The contraction shows that $a(h, y) \cos (\alpha(d) / 2) \leq a(x, y)$ for all $h \in H_{\mathrm{v}}$. Now the lemma follows from Corollary 6.3 .

The same reasoning proves the following.

6.5. Lemma. Let $y \in Y$ and assume a coefficient $b_{h} \geq 0$ is assigned to each $h \in H_{v}$. Suppose $y$ is not held by $Y \backslash\{x\}$. If $b_{x}<\frac{1}{2}(\cos (\alpha(d) / 2)) b_{h_{0}}$, for some $h_{0} \in H_{y}$, then $\left\|\sum b_{h} \mathbf{y h}\right\|>\frac{1}{2}(\cos (\alpha(d) / 2)) b_{h_{0}}$. 
6.6. Suppose $Y$ is approximately pointwise extremal, in the sense that it satisfies the hypotheses of Proposition 4.6. The results of Section 3 are still valid (with the exception of Lemma 3.3(1) and 3.4). So are the results of this section up to Lemma 6.4. Finally, Lemmas 6.4 and 6.5 remain true in view of the angle comparison Lemma 4.5 .

\section{Approximately Minimal Sets and the Implicit Function Theorem}

In Section 2 we described a computer procedure for obtaining minimal sets. The object of this section is to find estimates to justify that procedure. We will find conditions on an approximately minimal set which guarantee that there is a genuine minimal set nearby.

Since it is unknown whether every pointwise extremal set is minimal, we have to assume that the coefficients $a_{k}$ in equation $(* *)$ of Section 6 have the same sign. It follows by 6.6 and Lemma 6.4 that the coefficients are bounded away from 0 . Thus such a condition on the $a_{k}$ is computer-verifiable.

We also have to require a more stringent inequality than $4.6(3)$ on the maximal distances. This is because we use an implicit function-type argument, which is of a different kind than the results of the previous sections.

Suppose $Y \subset S^{2}$ has $n$ points and diam $Y<2 \pi / 3$. We order the set of all pairs of points of $Y$ by decreasing distance: $\operatorname{diam} Y=d_{1} \geq d_{2} \geq \cdots \geq d_{2 n-2} \geq d_{2 n-1}$, etc. By abuse of notation we will not distinguish between a pair of points and the distance between them. Choose $2 \pi / 3>d_{0}>d_{1}$. Let $\alpha(d)$ be defined as in 4.4 .

7.1. Proposition. Suppose a set $Y \subset S^{2}$ with diam $Y<2 \pi / 3$ satisfies the following three conditions:

1. $Y$ is separable.

2. There are numbers $b_{k} \geq 0, k=1, \ldots, 2 n-2$, such that, for every $y \in Y$, we have $\sum b(h, y) \mathbf{y h}=0$, where the summation is taken over all $h \in Y$ such that $\{h, y\} \in\left\{d_{k}\right\}_{k=1}^{2 n-2}$ and $b(h, y)=b_{k}$ if $\{h, y\}=d_{k}$.

3. $d_{1}-d_{2 n-2}<\lambda$, where

$$
\begin{gathered}
\lambda=\frac{1}{\sqrt{2 n-2(n-1) 2^{n-1}}}\left(\cos \frac{\alpha\left(d_{0}\right)}{2}\right)^{2 n-2} c \\
\text { and } c=\frac{1}{4} \min \left(d_{0}-d_{1}, d_{2 n-2}-d_{2 n-1}\right) \text {. }
\end{gathered}
$$

Then there is a minimal set $Y_{m}$ Hausdorff-near $Y$, i.e., $\operatorname{dist}_{\mathrm{H}}\left(Y, Y_{m}\right)<c$, whose pairs of points at maximal distance correspond to the pairs $d_{1}, \ldots, d_{2 n-2}$.

Note that the difference $d_{2 n-2}-d_{2 n-1}$ in the definition of $c$ is not essential as it can be bounded from below in terms of, say, $d_{0}-d_{1}$ (cf. 4.7). Note also that the affirmative answer to Question 1 of the Introduction would probably allow one to replace $7.1(3)$ with $4.6(3)$. 
Proof. Let $f: V \rightarrow R^{2 n-2}$ be given by coordinate functions $f_{1}, \ldots, f_{2 n-2}$ defined in Section 6. Then $f(Y)$ lies "near" the diagonal line $L \subset R^{2 n-2}$. Let $\pi: R^{2 n-2} \rightarrow L^{\perp}$ be the orthogonal projection to the complement of $L$. We will show that $\pi \circ f$ maps a ball of radius $c$ at $Y \in V$ (where $V$ is endowed with the Riemannian metric of the Cartesian product) onto a neighborhood containing a ball of radius $\sqrt{2 n-2} \lambda$ at $\pi \circ f(Y)$. This will show that all the $f_{k}$ take the same value at some $Y_{m} \in V$ because the distance from $f(Y)$ to $L$ is $<\sqrt{2 n-2}\left(d_{1}-d_{2 n-2}\right)$.

Condition 2 implies that each point of $Y$ is held by $\left\{d_{k}\right\}$ in the sense of the paragraph preceding Proposition 4.6. By 6.6 and Lemma 6.1, $b_{k}>0$. Since the ball $B(Y, c)$ at $Y \in V$ of radius $c$ is connected, it follows that $Y_{m}$ is a minimal set. This proves the proposition because dist $_{H} \leq$ dist $_{V}$.

We define the contracting coefficient of a linear map $g: R^{p} \rightarrow R^{q}$ to be

$$
\min _{v \in R^{i} \backslash\{0\}} \frac{\|g(v)\|}{\|v\|}
$$

Let $Z \in B(Y, c)$. We will estimate separately the contracting coefficients of $T_{Z} f$ (Lemma 7.2) and $\pi$ (Lemma 7.3) restricted to suitable subspaces. The proposition follows by multiplying these coefficients.

7.2. Lemma. Let $\left.\pi\right|_{\operatorname{Im} T f}$ be the restriction of $\pi$ to the image of Tf. Then the contracting coefficient of $\left.\pi\right|_{\operatorname{Im} \pi f}$ is $\geq\left(\cos \left(\alpha\left(d_{0}\right) / 2\right)\right)^{n-1}$.

Proof. Consider a slice for the action of $S O(3)$ on $V$. The map $f$ imbeds the slice in $R^{2 n-2}$. The image of $T f$ is parallel to the hyperplane $a_{1} x_{1}+\cdots+$ $a_{2 n-2} x_{2 n-2}=0$, where the $a_{k}$ are as in (**). The contracting coefficient equals the cosine of the angle between the normal vectors of $\operatorname{Im} \operatorname{Tf}$ and $L^{\perp}$ :

$$
\frac{a_{1}+\cdots+a_{2 n-2}}{\sqrt{2 n-2} \sqrt{a_{1}^{2}+\cdots+a_{2 n-2}^{2}}} \geq \frac{\min _{k} a_{k}}{\max _{k} a_{k}} \geq\left(\cos \frac{\alpha\left(d_{0}\right)}{2}\right)^{n-1} .
$$

The last inequality is true by 6.6 and Lemma 6.4 .

7.3. Lemma. The contracting coefficient $\delta$ of $\left.T f\right|_{N^{\perp}}$ satisfies

$$
\delta \geq \frac{1}{(n-1) 2^{n-1}}\left(\cos \frac{\alpha\left(d_{0}\right)}{2}\right)^{n-1} .
$$

Proof. We have

$$
\delta=\min _{v \in N^{\perp} \backslash\{0\}} \frac{\|T f(v)\|}{\|v\|} \geq \min _{v \in N^{\perp},\|v\|=1} \max _{k}\left|d f_{k}(v)\right| .
$$


Let $v \in N^{\perp} \subset T_{Z} V,\|v\|=1$. Let $\sigma: N^{\perp} \rightarrow v^{\perp}$ be the projection to the hyperplane $v^{\perp} \subset N^{\perp}$ orthogonal to $v$. By Caratheodory's theorem we may choose an index $k_{1}$ and numbers $b_{k} \geq 0$ so that $b_{k_{1}}=0$ and $\sum b_{k} \sigma\left(d f_{k}\right)=0$. Here $T V$ and $T^{*} V$ are identified using the Riemannian metric. Therefore $\sum b_{k} d f_{k}$ is proportional to $v$ and $\left\|\sum b_{k} d f_{k}\right\|=\left|\sum b_{k} d f_{k}(v)\right|$. Let $b_{k_{2}}$ be the biggest of the coefficients. Choose a sequence of $m$ covectors joining $d f_{k_{1}}$ with $d f_{k_{2}}$ as in Corollary 6.3. Relabel the covectors so that $k_{1}=1$ and $k_{2}=m$, and so the sequence becomes $d f_{1}, \ldots, d f_{m}$. Here $b_{1}=0$ and $m \leq n$.

If $0<\varepsilon<1$, let $i$ be the biggest index such that $\varepsilon b_{1}>b_{1-1}\left(i \geq 2\right.$ since $\left.b_{1}=0\right)$. Then $b_{k-1} / b_{k} \geq \varepsilon$ for all $k>i$, and

$$
b_{i}=\frac{b_{i}}{b_{i+1}} \times \cdots \times \frac{b_{m-1}}{b_{m}} b_{m} \geq \varepsilon^{m-1} b_{m} \geq \varepsilon^{m-2} b_{m} .
$$

Let $\varepsilon=\frac{1}{2} \cos \left(\alpha\left(d_{0}\right) / 2\right)$. Then by 6.6 and Lemma 6.5 we have $\left\|\sum b_{k} d f_{k}\right\| \geq \varepsilon b_{1} \geq$ $\varepsilon^{m-1} b_{m}$. Since $\left\|\sum b_{k} d f_{k}\right\|=\left|\sum b_{k} d f_{k}(v)\right|$, the largest summand $\left|b_{e} d f_{e}(v)\right|$ satisfies $\left|b_{e} d f_{e}(v)\right| \geq(1 /(m-1)) \varepsilon^{m-1} b_{m}$ (since $\left.b_{1}=0\right)$. But $b_{m} \geq b_{e}$, therefore $\left|d f_{e}(v)\right| \geq$ $(1 /(m-1)) \varepsilon^{m-1}$, and so also

$$
\delta \geq \frac{1}{m-1} \varepsilon^{m-1} \geq \frac{1}{(n-1) 2^{n-1}}\left(\cos \frac{\alpha\left(d_{0}\right)}{2}\right)^{n-1} .
$$

\section{References}

1. L. J. Billera and C. W. Lee, A proof of the sufficiency of McMullen's conditions for $f$-vectors of simplicial convex polytopes, J. Combin. Theory Ser. A 31 (1981), 237-255.

2. K. Borsuk, Drei Sätze über die $n$-dimensionale euklidische Sphäre, Fund. Math. 20 (1933), $177-190$.

3. J. Cheeger and D. G. Ebin, Comparison Theorems in Riemannian Geometry, North-Holland, Amsterdam, 1975.

4. L. Fejes Tóth, Lagerungen in der Ebene auf der Kugel und im Raum, Zweite Aufage, SpringerVerlag, Berlin, 1972.

5. M. Gromov, Curvature, diameter and Betti numbers, Comment. Math. Helv. 56 (1981), $179-195$.

6. M. Gromov, Filling Riemannian manifolds, J. Differential Geom., 18 (1983), 1-147.

7. K. Grove and K. Shiohama, A generalized sphere theorem, Ann. of Math. 106 (1977), $201-211$.

8. B. Grünbaum, A proof of Vázsonyi’s conjecture, Bull. Res. Council Israel A 6 (1956), 77-78.

9. A. Heppes, Beweis einer Vermutung von A. Vázsonyi, Acta Math. Acad. Sci. Hungar. 7 (1956), 463-466.

10. M. Katz, The filling radius of two-point homogeneous spaces, J. Differential Geom. 18 (1983), $505-511$.

11. L. Lovász, Self-dual polytopes and the chromatic number of distance graphs on the sphere, Acta Sci. Math. 45 (1983), 317-323.

12. P. McMullen, The numbers of faces of simplicial polytopes, Israel J. Math. 9 (1971), 559-570.

13. P. McMullen and G. C. Shephard, Convex Polytopes and the Upper Bound Conjecture, London Mathematical Society Lecture Note Series, Vol. 3, Cambridge University Press, London, 1971. 
14. J. Molnár, Über eine Übertragung des Hellyschen Satzes in sphärische Räume, Acta Math. Acad. Sci. Hungar. 8 (1957), 315-318.

15. R. Stanley, The number of faces of a simplicial convex polytope, Adv. in Math. 35 (1980), $236-238$.

16. S. Straszewicz, Sur un problème géométrique de P. Erdös, Bull. Acad. Polon. Sci. Cl. III 5 (1957), 39-40.

17. D. W. Walkup, The lower bound conjecture for 3- and 4-manifolds, Acta Math. 125 (1970), $75-107$.

Received September 3, 1986. 